dren, and allergic children who acquired tolerance to peanuts. J. Clin. Invest. 111:1065-1072. doi:10.1172/JCI200316142.

7. Cohen, S.B., Webb, L.M., and Feldmann, M 1996. The method of deriving human T-cell clones alters the proportion of IL-10-producing cells. Immunology. 87:343-347.

8. Demeure, C.E., et al. 1995. Human naive CD4 T cells produce interleukin-4 at priming and acquire a Th2 phenotype upon repetitive stimulations in neutral conditions. Eur. J. Immunol. 25:2722-2725.

9. Valentine, M.D., et al. 1990. The value of immunotherapy with venom in children with allergy to insect stings. N. Engl. J. Med. 323:1601-1603

10. Oppenheimer, J.J., Nelson, H.S., Bock, S.A., Christensen, F., and Leung, D.Y. 1992. Treat- ment of peanut allergy with rush immunotherapy. J. Allergy Clin. Immunol. 90:256-262.

11. Burks, A.W., Bannon, G.A., and Lehrer, S.B. 2001. Classic specific immunotherapy and new perspectives in specific immunotherapy for food allergy. Allergy. 67:121-124.

12. Skolnick, H.S., et al. 2001. The natural history of peanut allergy. J. Allergy Clin. Immunol. 107:367-374.

\title{
Heparan sulfate: Antithrombotic or not?
}

\author{
Jeffrey I. Weitz \\ Henderson Research Centre, Hamilton, Ontario, Canada \\ J. Clin. Invest. 111:952-954 (2003) doi:10.1172/JCI200318234.
}

Tissue factor exposed at sites of vascular injury initiates coagulation resulting in thrombin generation. In addition to converting fibrinogen to fibrin, thrombin amplifies its own generation by activating platelets and by activating factors $\mathrm{V}$ and VIII, key cofactors in coagulation. Consequently, tight regulation of thrombin activity is essential to prevent excessive thrombosis (1).

\section{Regulation of thrombin}

Two naturally occurring anticoagulant pathways serve to regulate thrombin; the protein $\mathrm{C}$ pathway and antithrombin. The protein $C$ pathway is initiated when thrombin binds to thrombomodulin, a thrombin receptor expressed by endothelial cells (2). Once bound to thrombomodulin, thrombin undergoes a conformational change at its active site that converts it from a procoagulant enzyme into a potent activator of protein C. Activated protein $\mathrm{C}$, in concert with its cofactor protein $S$, serves as an anticoagulant by degrading and inactivating activated factors V and VIII (2).

Several lines of evidence highlight the physiological importance of the

\footnotetext{
Address correspondence to: Jeffrey I. Weitz, Henderson Research Centre, 711 Concession Street, Hamilton, Ontario L8V 1C3, Canada. Phone: (905) 574-8550; Fax: (905) 575-2646; E-mail: jweitz@thrombosis.hhscr.org. Conflict of interest: The author has declared that no conflict of interest exists.

Nonstandard abbreviations used: serine proteinase inhibitor (serpin); 3-O-sulfotransferase-1 (3-OST-1).
}

protein $\mathrm{C}$ pathway. Mice totally deficient in thrombomodulin die in utero (3), whereas ablation of endothelial thrombomodulin causes early onset thrombosis (4). Likewise, patients with protein $\mathrm{C}$ or protein $\mathrm{S}$ deficiency are prone to thrombosis, as are those with the factor $V$ Leiden mutation, a point mutation that renders activated factor $\mathrm{V}$ Leiden relatively resistant to inactivation by activated protein $C(5)$.

Antithrombin also is critical for the regulation of coagulation. A member of the serine proteinase inhibitor (serpin) superfamily, antithrombin inhibits thrombin and other clotting enzymes in a slow, progressive fashion. The importance of antithrombin is highlighted by the fact that patients with heterozygous antithrombin deficiency have a thrombotic tendency $(5,6)$. Complete deficiency of antithrombin is likely to be incompatible with life, a concept supported by the observation that knocking out the antithrombin gene in mice results in intrauterine death from massive thrombosis (7).

\section{Heparan sulfate}

The activity of antithrombin is enhanced by heparin. Antithrombin possesses a heparin binding site that interacts with a unique pentasaccharide sequence found on one-third of the chains of unfractionated heparin. Key to high-affinity binding of antithrombin to this pentasaccharide sequence is 3-O-sulfated glucosamine, the middle saccharide unit of the pentasaccharide (8). Pentasaccharide binding to antithrombin induces conformational changes in the reactive center loop of the serpin that accelerate the rate at which antithrombin inhibits its target proteases by two to three orders of magnitude.

Although heparin is found in granules of human mast cells, it is not the physiological counterpart of medicinal heparin. Recent studies indicate that mast cell heparin regulates the types and amounts of positively-charged proteases stored in mast cell granules. Thus, mast cells deficient in heparinsynthesizing enzyme have altered morphology (9) and their granules contain reduced amounts of tryptase, chymase, and carboxypeptidase A (10). Not only are protease levels reduced, but their activity may also be limited because heparin enhances the activity of some mast cell proteases (11).

Current thinking is that the physiological counterpart of medicinal heparin is heparan sulfate, a glycosaminoglycan found on the surface of most eukaryotic cells and in the extracellular matrix. Anchored to the cell surface by its proteoglycan core, the functional units of heparan sulfate are found on its branching glycosaminoglycan side-chains (Figure 1). Cultured endothelial cells synthesize heparan sulfate and $1 \%$ to $10 \%$ of the molecules have anticoagulant activity because they contain the 3-O-sulfated glucosamine residue that is the hallmark of the antithrombin-binding pentasaccharide (11).

Antithrombin binds to cultured endothelial cells and binding is reduced when the cells are pretreated with heparinase (12), suggesting that this interaction is mediated by heparan sulfate. When radiolabeled antithrombin is used, over $90 \%$ of the antithrombin that binds to cultured endothelial cells or to the surface of perfused aortic segments can be localized to the subendothelial matrix (13). Exposure of cultured endothelial cells to IL-1 or tumor necrosis factor reduces heparan sulfate 


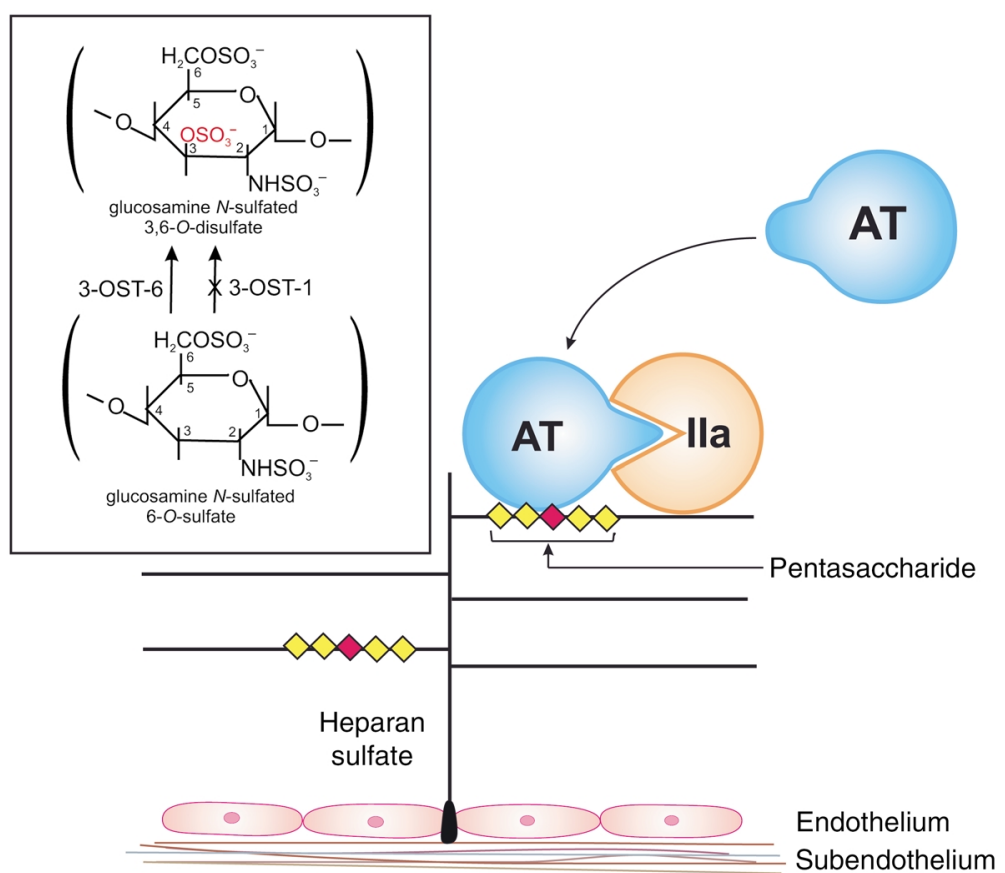

Figure 1

Heparan sulfate anticoagulant pathway. Anchored by a proteoglycan core to the vessel wall, heparan sulfate binds antithrombin (AT) via a unique pentasaccharide sequence (diamonds) found on 1 to $10 \%$ of the glycosaminoglycan side-chains. Key to the high-affinity binding of AT to the pentasaccharide is the 3-O-sulfated glucosamine residue in the middle of the pentasaccharide sequence (red diamond). Once bound, AT undergoes a conformational change at its reactive centre loop that enhances its reactivity with thrombin (Ila), thereby promoting the formation of AT/Ila complexes. Targeted disruption of 3-O-sulfotransferase-1 (3-OST-1) attenuates 3-O-sulfation (in red) of the critical glucosamine residue within the pentasaccharide sequence of heparan sulfate, thereby reducing its capacity to bind AT (inset). Antithrombin affinity is not abolished, however, because 3-OST-6, another 3-O-sulfotransferase isoform, can also generate 3-O-sulfated glucosamine residues.

biosynthesis (14), raising the possibility that subendothelial heparan sulfate may compensate for this phenomenon and prevent thrombosis when endothelial cells are activated or damaged.

\section{Heparan sulfate-deficient mice}

To explore the physiological importance of heparan sulfate, HajMohammadi and colleagues (15) generated mice deficient in 3-O-sulfotransferase-1 (3-OST-1), the enzyme primarily responsible for 3-O-sulfation of the glucosamine residue within the pentasaccharide sequence. As reported in this issue of the JCI, tissue extracts from these mice exhibit reduced capacity to bind antithrombin and to enhance its reaction with factor Xa compared with extracts from tissues of wild-type mice. Despite reduced heparan sulfate activity, the knockouts have a normal phenotype and have the same amount of fibrin deposition in their tissues as wild-type mice under normoxic and hypoxic conditions. of redundancy in hemostatic pathways, one designed to limit thrombin generation at sites of vascular injury.

\section{Is heparan sulfate antithrombotic?}

Do the findings in the 3-OST-1 knockout mice indicate that heparan sulfate is not antithrombotic? I think not, based on experiments in nature. Antithrom$\operatorname{bin}_{\text {Toyoma }}(18)$ and antithrombin Fontainbleu (19) are examples of congenital antithrombin variants that react normally with thrombin in the absence of heparin, but fail to show accelerated inhibition when heparin is present. The lack of a heparin effect can be explained by the fact that these variants have reduced affinity for heparin because of mutations in their heparin-binding domain. Only patients who are homozygous for these variant antithrombins are prone to unprovoked thrombosis, suggesting that dysregulation of thrombin inhibition requires complete disruption of the antithrombin-heparan sulfate interaction. Supporting this concept are the findings in mice that have been engineered to express antithrombin with reduced capacity to bind heparan sulfate. Homozygotes die of thrombosis early in life, whereas heterozygotes have a normal phenotype (20). Thus, only complete deficiency of heparan sulfate leads to thrombosis.

How do we rationalize the unprovoked thrombosis that occurs in humans or mice homozygous for antithrombin mutations that reduce antithrombin's affinity for heparan sulfate, with the absence of thrombosis in the 3-OST-1 knockout mice? The answer to this question may lie in the details of the study. Knocking out 3-OST-1 attenuates, but does not abolish, the ability of heparan sulfate to bind and activate antithrombin. As the authors indicate, the residual 2 to $20 \%$ heparan sulfate activity in the 3-OST-1 knockouts likely reflects 3 - $O$-sulfation by 3-O-sulfotransferase isoforms other than 3-OST-1, such as 3-OST-6. This residual heparan sulfate activity may be sufficient to protect the mice from thrombosis as is the case in patients heterozygous for mutations in the heparin-binding domain of antithrombin. Testing this possibility requires knockout mice with no heparan sulfate activity. Likely, the generation of such mice will require targeted disruption of several 3-OST isoforms. Based on results of experiments 
in nature, I predict that mice with no heparan sulfate activity will develop thrombosis, supporting the concept that heparan sulfate is antithrombotic. These mice also should exhibit more profound intrauterine growth retardation than 3-OST-1 knockouts (15), thereby demonstrating a biological gradient for this unexpected finding depending on the extent to which heparan sulfate activity is reduced.

\section{Summary}

With their studies in 3-OST-1 knockout mice, HajMohammadi and colleagues (15) have advanced our understanding of the pathways that regulate thrombin. Antithrombin is critical for thrombin regulation because even partial deficiency is associated with thrombosis. In contrast, complete deficiency of heparan sulfate activity is necessary to provoke thrombosis. These findings suggest that small amounts of residual heparan sulfate activity are sufficient to catalyze antithrombin or that other vessel wall glycosaminoglycans can compensate for all but complete lack of heparan sulfate activity.

\section{Acknowledgments}

J.I. Weitz is the recipient of a Career Investigator Award from the Heart and Stroke Foundation of Ontario and holds the Heart and Stroke Foun- dation of Ontario/J. Fraser Mustard Chair in Cardiovascular Research, and the Canada Research Chair in Thrombosis at McMaster University.

1. Mann, K.G., Butenas, S., and Brummel, K. 2003. The dynamics of thrombin formation. Arterioscler. Thromb. Vasc. Biol. 23:17-25.

2. Esmon, C.T., et al. 1997. The protein C pathway: new insights. Thromb. Haemost. 78:70-74.

3. Healy, A.M., Rayburn, H.B., Rosenberg, R.D., and Weiler, H. 1995. Absence of the blood-clotting regulator thrombomodulin causes embryonic lethal ity in mice before development of a functional cardiovascular system. Proc. Natl. Acad. Sci. U. S. A. 92:850-854

4. Isermann, B., et al. 2001. Endothelium-specific loss of murine thrombomodulin disrupts the protein $\mathrm{C}$ anticoagulant pathway and causes juvenileonset thrombosis. J. Clin. Invest. 108:537-546. doi:10.1172/JCI200113077.

5. Thomas, D.P., and Roberts, H.R. 1997. Hypercoagulability in venous and arterial thrombosis. Ann. Intern. Med. 126:638-644.

6. van Boven, H.H., and Lane, D.A. 1997. Antithrombin and its inherited deficiency states. Semin. Hematol. 34:188-204.

7. Ishiguro, K., et al. 2000. Complete antithrombin deficiency in mice results in embryonic lethality. J. Clin. Invest. 106:873-878.

8. Atha, D.H., Lormeau, J.C., Petitou, M., Rosenberg, R.D., and Choay, J. 1987. Contribution of 3-Oand 6-O-sulfated glucosamine residues in the heparin-induced conformational change in antithrombin III. Biochemistry. 26:6454-6461.

9. Forsberg, E., et al. 1999. Abnormal mast cells in mice deficient in a heparin-synthesizing enzyme. Nature. 400:773-776

10. Humphries, D.E., et al. 1999. Heparin is essential for the storage of specific granule proteases in mast cells. Nature. 400:769-772.

11. Hallgren, J., Spillmann, D., and Pejler, G. 2001. Structural requirements and mechanism of heparin-induced activation of a recombinant mouse mast cell tryptase, mouse mast cell pro- tease-6: formation of active tryptase monomers in the presence of low molecular weight heparin. J. Biol. Chem. 276:42774-42781.

12. Marcum, J.A., et. al. 1986. Cloned bovine aortic endothelial cells synthesize anticoagulantly active heparan sulfate proteoglycan. J. Biol. Chem. 261:7507-7517.

13. de Agostini, A.I., Watkins, S.C., Slayter, H.S., Youssoufian, H., and Rosenberg, R.D. 1990. Localization of anticoagulantly active heparan sulfate proteoglycans in vascular endothelium: antithrombin binding on cultured endothelial cells and perfused rat aorta. J. Cell. Biol. 111:1293-1304.

14. Kobayashi, M., Shimada, K., and Ozawa, T. 1990 Human recombinant interleukin-1 beta- and tumor necrosis factor alpha-mediated suppression of heparin-like compounds on cultured porcine aortic endothelial cells. J. Cell. Physiol. 144:383-390.

15. HajMohammadi, S., et al. 2003. Normal levels of anticoagulant heparan sulfate are not essential for normal hemostasis. J. Clin. Invest. 111:989-999. doi:10.1172/JCI200315809.

16. Nawa, K., et al. 1990. Presence and function of chondroitin-4-sulfate on recombinant human soluble thrombomodulin. Biochem. Biophys. Res. Commun. 171:729-737.

17. Koyama, T., et al. 1991. Different glycoforms of human thrombomodulin. Their glycosaminoglycan-dependent modulatory effects on thrombin inactivation by heparin cofactor II and antithrombin III. Eur. J. Biochem. 198:56-70.

18. Koide, T., Odani, S., Takahashi, K., Ono, T., and Sakuragawa, N. 1984. Antithrombin III Toyama: replacement of arginine- 47 by cysteine in hereditary abnormal antithrombin III that lacks heparin-binding ability. Proc. Natl. Acad. Sci. U. S. A. 81:289-293.

19. Boyer, C., Wolf, M., Vedrenne, J., Meyer, D., and Larrieu, M.J. 1986. Homozygous variant of antithrombin III: AT III Fontainbleau. Thromb. Haemost. 56:18-22.

20. Dewerchin, M., et al. 2000. Life-threatening thrombosis in mice with targeted Arg 47 to Cys mutation of the heparin binding domain of antithrombin. Circulation. 104:351 (Abstr.).

\section{Transplacental thyroxine and fetal brain development}

\section{R. Thomas Zoeller}

Department of Biology, University of Massachusetts-Amherst, Morrill Science Center, Amherst, Massachusetts, USA

J. Clin. Invest. 111:954-957 (2003). doi:10.1172/JCI200318236.

Address correspondence to: $\mathrm{R}$. Thomas Zoeller, Department of Biology, University of Massachusetts-Amherst, Morrill Science Center, Amherst, Massachusetts 01003, USA. Phone: (413) 545-2088; Fax: (413) 545-3243;

E-mail: tzoeller@bio.umass.edu.

Conflict of interest: The author has declared that no conflict of interest exists.

Nonstandard abbreviations used: congenital hypothyroidism $(\mathrm{CH})$; intelligence quotient (IQ); thyroxine (T4); triiodothyronine (T3); disabled homolog 1 (Dab1).
We all know that thyroid hormone is essential for normal brain development, so it is perhaps not surprising to read the title of the report by Lavado-Autric et al. in this issue of the JCI (1), indicating that thyroid hormone affects brain development in the rat. However, the article addresses two matters that are central to an ongoing debate about important details of thyroid hormone action in the developing brain.

Lavado-Autric et al. (1) report that subtle insufficiency of thyroid hormone in the pregnant rat disrupts the migration of neurons in the fetal cortex and hippocampus, leading to the presence of neurons in aberrant locations of the adult offspring's brain and "blurring" cortical layers. Thus, the two important issues addressed in the design of this work are the timing of thyroid hormone action in the developing brain and the relative sensitivity of the fetal brain to maternal thyroid hormone insufficiency. Considering that maternal hypothyroxinemia may be 150-200 times more prevalent than congenital hypothyroidism $(\mathrm{CH})$, several authors have recently speculated that screening for thyroid function should be routine for women early in their pregnancy 